\title{
VARIATION OF THE ENTROPY OF THE TRANSITION IN NEMATIC MIXTURES
}

Nagappa , K. N. Jagadish , J. Mahadeva , S. K. Naveenkumar \& R. Somashekar

To cite this article: Nagappa , K. N. Jagadish , J. Mahadeva, S. K. Naveenkumar \& R. Somashekar (2001) VARIATION OF THE ENTROPY OF THE TRANSITION IN NEMATIC MIXTURES, Molecular Crystals and Liquid Crystals Science and Technology. Section A. Molecular Crystals and Liquid Crystals, 366:1, 239-246, DOI: 10.1080/10587250108023966

To link to this article: https://doi.org/10.1080/10587250108023966

曲 Published online: 24 Sep 2006.

Submit your article to this journal $\pi$

Щ Article views: 19

Q View related articles $\sqsubset$

Citing articles: 2 View citing articles ¿ð 


\title{
Variation of the Entropy of the Transition in Nematic Mixtures
}

\author{
NAGAPPA, K.N. JAGADISH, J. MAHADEVA, S.K. NAVEENKUMAR \\ and R. SOMASHEKAR.
}

Department of Studies in Physics, University of Mysore, Manasagangotri, Mysore 570 006, INDIA

The temperature variation of orientational order parameter in binary mixtures of two nematic compounds viz $\mathrm{P}$-(p-ethoxy-phenylazo) phenyl Valerate (EPPV) and $\mathrm{N}$-(p-propoxybenzylidene)p-pentylaniline (PBPA) has been estimated using refractive index and density data. The higher order parameter $\left\langle\mathrm{p}_{4}\right\rangle$ has been estimated. Employing the Humphries-James-Luckhurst theory the entropy of the transition of the mixtures at different concentrations has been computed. It is observed that the increase in the value of entropy of the transition is associated with the increase in the pair potential of the molecules in the mixtures.

Keywords: Order parameter; Pair potential; Entropy

\section{INTRODUCTION}

Namatic liquid crystals are characterised by the presence of long range orientational order of rod-like molecules. Nematic phase is the least orderd of all liquid crystalline phase. The orintational order parameter is computed from anistropic properties such as 
refractive index, elastic constant, magnetic susceptibility, etc of the nematic phase.

Mixture of nematic liquid crystals has a wide application in display devices due to their exquisite optical properties ${ }^{[1]}$, such nematic mixtures are stable, chemically as well as photochemically ${ }^{[2,3]}$. By mixing two nematogens one can often obtain a material with a lower melting point. Usually two nematogens exhibit the property of continous miscibility without crossing any line (first or second order transition line) and posses the same symmetry ${ }^{[1]}$. This miscibility situation is true not only between usual nematogens but also between nematic and cholesteric esters. By mixing two nematogens, chistyakov ${ }^{[4]}$ has observed the extension of the mesomorphic range. Binary systms offer the advantages of frequetly exhibiting eutectic behaviours in their solid-mesophase transition, whereas the mesophase-isotropic transition temperature varies linearly with composition. In particular, several binary nematic systems have been studied ${ }^{[5]}$ and only small deviations from linearity in the variation of nematic-isotroic transition temperature are noted, even in cases with significant differences in molar volumes and densities of the components. This general behaviour has been satisfactorally accounted for theoretically by Humphries and Luckhurst ${ }^{[6]}$.

Higher nematic liquid crystal phase transition decrease the resistance of the liquid crystal and hence increase in the higher power consumption in an active matrix addressed liquid crystal display. There is a continued interest in the nematic liquid crystals due to their wide range of exquisite optical and physical properties ${ }^{[7]}$. One such property is the orientational order, which determines the applicability of the nematics in electro-optic devices or displays. To explain the temperature dependance of the orientational order pa 
rameter, the molecular field theory of nematic using anisotropic dispersion forces was given by Maier and Saupe ${ }^{[8]}$ which is essentially an extension of London theory ${ }^{[9,10]}$. Humphries-James-Luckhurst ${ }^{[11]}$ have determined the following expression based on averaging of the anisotropic pair potential over the co-ordinates of one particle, which we find very easy to compute and they are

$$
<P_{L}>=\frac{1}{Z} \int d \beta \sin \beta P_{L}(\cos \beta) f(\beta)
$$

where the orientational distribution function

$$
f(\beta)=\exp \left(-\beta \sum_{L=1} \overline{u_{L}} \overline{P_{L}} P_{L}(\cos \beta)\right)
$$

and orientational partition

$$
Z=\int d \beta \sin \beta f(\beta)
$$

The entropy of the transition, after truncating the summation to two terms, is given by

$$
\frac{\Delta S}{N k}=\frac{N}{2}\left[\frac{\bar{u}_{2}}{k T_{N I}}\right]\left({\overline{p_{2}}}^{2}+\lambda{\overline{p_{4}}}^{2}\right)
$$

In this paper, we have determined $\left\langle p_{2}\right\rangle,\left\langle p_{4}\right\rangle, \frac{\mathbb{u}_{2}}{-k T_{N 1}}$ and $\frac{\Delta S}{N k}$ for the nematic mixtures and the results are presented accordingly.

\section{Experimental:}

The nematic compounds used in the investigation are $\mathrm{P}$-(pethoxy phenylazo)phenyle valerate (EPPV) and $\mathrm{N}$-(p-propoxy benzeyledene)p-pentylaniline (PBPA) which are a schiff base. These samples were obtained from M/S Eastman organic chemicals, USA the structural formulae and transition temperatures of these nematic compounds are shown in Fig.1. Mixtures of EPPV in PBPA 
of different concentrations were prepared. The components of mixtures were well mixed in the molten state and allowed to cool very slowly. The transition temperatures of the mixtures determined using Leitz polarizing microscope and a hot stage. The values of phase transition temperatures were found to be in good agreement with that determined by differential scanning calorimetry using PerkinElmer DSC-2.

It is well known that binary mixtures of liquid crystals exhibit a pronounced minimum in their clearing temperature as a function of concentration as shown in Fig. 2. The densities of mixtures at different temperatures were determined by measuring the length of a pellet of known mass of the spicemen taken in a calibrated capillary tube. The values of densities are accurate to $0.001 \mathrm{gm} / \mathrm{cc}$. Refractive indeces of $n_{\mathrm{e}}$ and $n_{\mathrm{o}}$ of the mixtures were determined at different temperatures using $A b b e$ refractometer and Goniometer spectrometer for the wavelength $5893 \AA$.

\section{Computation}

Employing Neugebauer relations and using the refractive index and density data ,orientational order parameter $\left\langle p_{2}\right\rangle$ has been determined for all the mixtures and this has been reported partly in our earlier paper ${ }^{[12,13]}$. Using the equation of Humphries-JamesLuckhurst we have carriedout the computation for all the mixtures and we find that the orientational order parameter curve with temperature fits very well for $\lambda=0.01$ and the corresponding values of $\frac{\hat{q}_{2}}{-k T_{N I}}$ are given in Figure as a function of temperature and concentration .

From these we have re-computed $\left\langle p_{2}\right\rangle$ and hence estimated $\left\langle p_{4}\right\rangle$ the higher order parameter. Employing the relation (4), we have estimated the entropy of the transition for all the mixtures 
and these are tabulated in Table 1 along with the values of $\left\langle p_{2}\right\rangle$ and $\left\langle p_{4}\right\rangle$ determined near the transition. Figures $3(a-c)$ give the variation of $\frac{a_{2}}{-k T_{N 1}}$, $\left.<p_{2}\right\rangle$ and $\left\langle p_{4}\right\rangle$ with concentration and temperature for the mixture.

Table 1. Entropy and Order Parameter of Mixture EPPV in PBPA

$$
\left.T_{c} \text { Concentration } \frac{\varepsilon_{2}}{-k T_{N I}}<p_{2}\right\rangle\left\langle p_{4}\right\rangle \quad \frac{\Delta S}{N k}
$$

$\begin{array}{cccccc}122^{\circ} \mathrm{C} & 10 \% \text { PBPA } & 4.531 & 0.3647 & 0.0866 & 0.3003 \\ 110^{\circ} \mathrm{C} & 30 \% \text { PBPA } & 4.557 & 0.3706 & 0.0894 & 0.3119 \\ 98^{\circ} \mathrm{C} & 50 \% \text { PBPA } & 4.559 & 0.3766 & 0.0923 & 0.3223 \\ 86^{\circ} \mathrm{C} & 70 \% \text { PBPA } & 4.535 & 0.3766 & 0.0923 & 0.3206 \\ 81^{\circ} \mathrm{C} & 80 \% \text { PBPA } & 4.549 & 0.3797 & 0.0938 & 0.3268 \\ 75^{\circ} \mathrm{C} & 90 \% \text { PBPA } & 4.564 & 0.3828 & 0.0954 & 0.3333\end{array}$

\section{Result and discussion}

From Figures 3(a-c) important behavior to be noticed are the following.

1. With increase in concentration $\left\langle p_{2}\right\rangle$ as well as $\left\langle p_{4}\right\rangle$ increases indicating that the pseudo potential and hence the anisotropic pair potential becomes steeper with the additions of PBPA.

2. This is also justified by the systematic increase in the values of the entropy of the transition in this mixed system.

3. However, it should be noted from Table.1, that, the increase in the entropy of the transition is associated with the decrease in 
the transition temperature for the corresponding increase in the concentration. This is quite understanding in terms of the fact that the anisotropic pair potential becornes steeper with increase in the concentration of EPPV in PBPA.

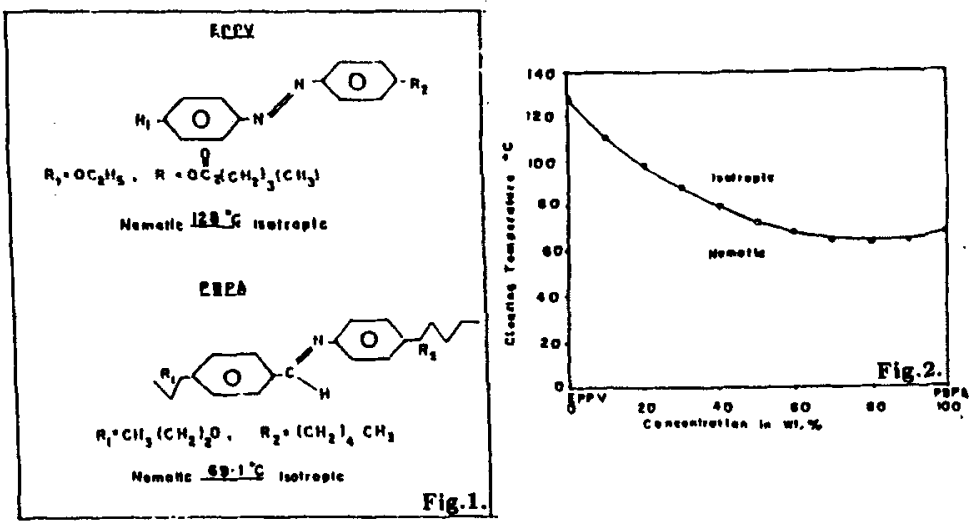

Fig.1. Structural formulae and transition temperatures of EPPV and PBPA

Fig.2. Clearing temperatures as a function of $\mathrm{Wt} \%$ of EPPV in PBPA

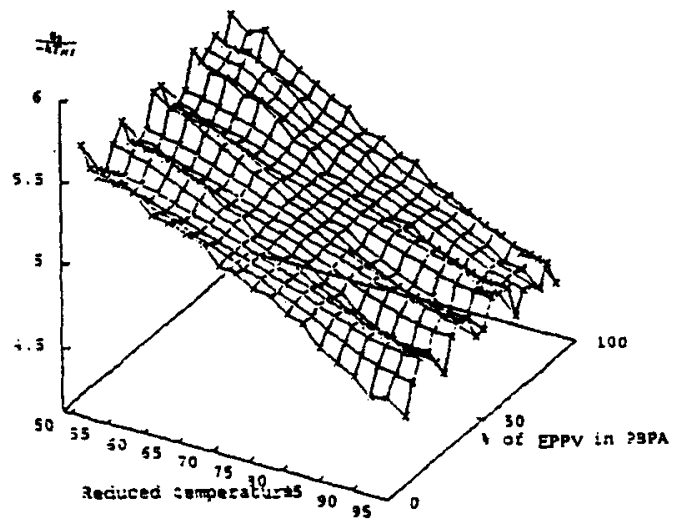

Fig.3(a) Variation of anisotropic pair potential with temperature and concentration 


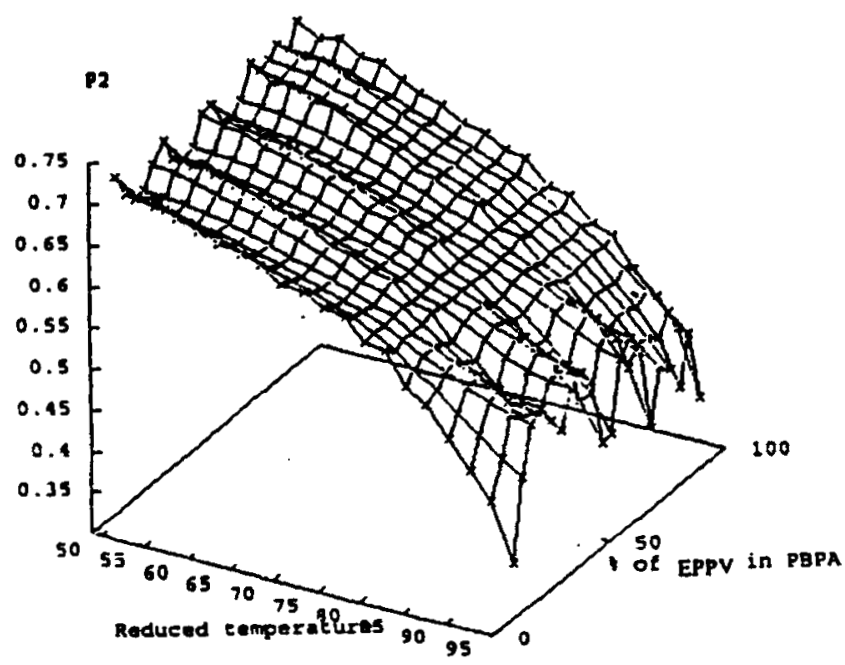

Fig.3(b) Variation of order parameter with temperature and concentration

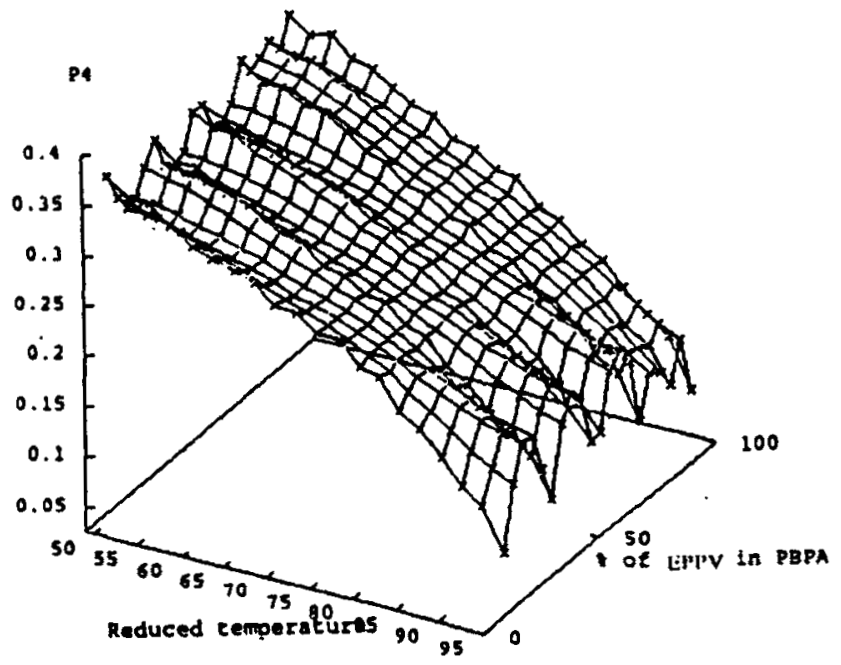

Fig.3(c) Variation of higher order parameter with temperature and concentration 


\section{Acknowledgments}

One of us J Mahadeva thank the University Grant Comission (UGC), New Delhi, for awarding Teacher Fellow-FIP and financial support.

\section{References}

[1] P.G. de Gennes, The Physica of liquid crystal, Clarendon press, oxford (1974).

[2] B. Bahadur Mol. Cryst. Liq. Cryst, 109, 1-98 (1984).

[3] B. Bahadur, Editor, Liquid Crystals applications and uses, Vol. 1, World scientific Singapore, New York, (1990).

[4] I.G. Chistyakov, Soviet Physics Uspekhi, 9, 551 (1967).

[5] S. Chandrashekar and N.V. Madhusudana, J. de Phys. C4., 30, 24 (1969).

[6] R.L. Humphries, and G.R. Luckhurst, Chem. Phys. Lett, 23, 667 (1973).

[7] G.R. Luckhurst and G.W. Gray, The Molecular physics of liquid crystals (Eds) (London, NewYork, Academic Press) 85 (1979).

[8] W. Maier and A. Saupe, Z. Naturforsch., 13a, 564 (1958); 14a, 882 (1959); 15a, 287 (1960).

[9] F. London, Z. Physik., 63, 245 (1930).

[10] F. London, Z. Physik. Chem., 11B, 221 (1930).

[11] R.L. Humphries, P.G. James and G.R. Luckhurst, J. Chem. Soc.Faraday Trans. II 68, 1031 (1972).

[12] R. Somashekar, D. Revannasiddaiah, M.S. Madhava, and D. Krishnamurti, Mol. Cryst. Liq. Cryst., 45, 243 (1978).

[13] Nagappa, J. Mahadeva, H. Somashekarappa and R. Somashekar Indian J. Phys., 74A(1), 45 (2000). 